\title{
A De Gioannis, D De Leo
}

Oral Ketamine augmentation for chronic suicidality in treatment resistant depression.

To the editor: In a recent article in this journal the effectiveness of IV Ketamine in treatment resistant depression was highlighted (1). The following case reports describe the use of oral ketamine as augmentation treatment in patients presenting with chronic suicide ideation and at least two significant past suicide attempts.

Mr.B was a 44-year-old man with a history of bipolar depression and chronic suicide ideation since adolescence. His condition was further complicated by severe chronic pain and a family history of suicide. Despite multiple pharmacotherapy combinations, and the current regime of Amytryptiline (200mg nocte) and Quetiapine (100mg b.i.d.), he remained depressed, with scores of 36 on the MADRS and 4/6 on the suicide item. Following written informed consent, oral ketamine was added. The treatment involved fortnightly doses of a ketamine solution $(100 \mathrm{mg} / \mathrm{ml})$ ingested orally with a flavoured drink. Starting with an initial dose of $0.5 \mathrm{mg} / \mathrm{kg}$ and gradually increasing by $0.5 \mathrm{mg} / \mathrm{kg}$ with each treatment, we achieved sustained clinical response at around $3 \mathrm{mg} / \mathrm{kg}$ without any adverse or side effects. Within 24-hour from his first treatment, his score on MADRS and suicide item decreased to 17 and 1, respectively. Repeated treatments every two to three weeks produced sustained remission of his suicidal ideation.

Mrs.A was a 37-year-old woman with bipolar depression and suicide ideation since adolescence. Her medication history included adequate trials of Venlafaxine, Mirtazapine, Fluoxetine, Quetiapine, Olanzapine and several courses of ECT. Her current regime included Venlafaxine (150mg q.d.) and Quetiapine (700mg q.d.). She remained depressed with score on MADRS and suicidality of 31 and 4 respectively. After obtaining written informed consent, oral Ketamine was added. The initial dose of $0.5 \mathrm{mg} / \mathrm{kg}$ was gradually increased to $1.5 \mathrm{mg} / \mathrm{kg}$. Within 24 hours from her first treatment the scores 
decreased to 10 on the MADRS and 2 on the suicide item. She continued to receive monthly doses of oral ketamine and her mental state continued to improve with no suicide ideation between treatments.

Pre-treatment blood tests included liver function tests and CBC with differential. Blood pressure and pulse rate were monitored before and 30 minutes after each dose. Neither patient experienced adverse events or significant changes in vital signs during the treatment.

Although case reports require cautious consideration, these results are consistent with recent findings supporting the use of ketamine in treating severe depression. The possibility of using oral ketamine as a viable alternative to IV ketamine infusion is of special interest.

1. Katalinic N, Lai R, Somogyi A, Mitchell PB, Glue P and Loo CK (2013) Ketamine as a new treatment for depression: A review of its efficacy and adverse effects. Australian and New Zealand Journal of Psychiatry 47(8): 710-727. 